(evidence based) guidelines for oncological and palliative care. we use the unique combination of data from our national cancer registry, our knowledge and experience as well as the knowledge and experience of medical professionals and patient representatives available through national and regional networks. As part of this process, each evidence based guideline is evaluated with the objective: 1 . to get insight in the adherence; 2 . to get insight in possible bottlenecks for the implementation in daily practice; 3. to stimulate further implementation by giving feedback.

Description of Best Practice The case of the guideline renal cell carcinoma will be presented. This includes the following steps: 1. selection of recommendations (based on the new guideline) by the guideline working group; 2 . definition of indicators; 3 . registration by trained registration staff of our national cancer registry; 4. analysis of the results; 5. presentation of results to professionals; 6 . specific promotion of expertise and skills to further implement the guideline.

Lessons for Guideline Developers/Users The combination of development, implementation and evaluation of guidelines 'in one hand' offers special opportunities for quality improvement. During guideline development it already becomes clear which recommendations will need extra attention during implementation. These recommendations are very interesting to evaluate, so that the feedback about these recommendations can help the professionals to further implement the guideline.

\section{P041 SUSTAINING ORGANIZATIONAL CAPACITIES OF KNOWLEDGE UTILIZATION: A COMPLETE TOOLBOX FOR THE HEALTH AND SOCIAL SERVICES ORGANIZATIONS}

${ }^{1} \mathrm{~F}$ Chagnon, ${ }^{1} \mathrm{C}$ Bardon, ${ }^{1} \mathrm{~N}$ Houlfort, ${ }^{2} \mathrm{R}$ Proulx, ${ }^{1} \mathrm{P}$ Labelle, ${ }^{1} \mathrm{M}$ Gervais. ${ }^{1}$ Université $d u$ Québec a Montréal, Montreal, Canada; ${ }^{2}$ Centre Réadaptation Déficience Intelectulle Montréa (CRDI), Montreal, Canada

\section{0:1136/bmjqs-2013-002293.135}

Background Despite research and funding, the understanding of organization's capacities of knowledge utilisation remains incomplete in health and social field.

Objectives To better understand organisational determinants of research result utilisation.

Method Between 2009 and 2012, managers (n = 75) from health and social services organisations $(\mathrm{N}=8)$ have actively contributed in a participative research action process. Additionally professionals and managers $(\mathrm{N}=2161)$ have answered questionnaires. Qualitative and quantitative data gathered on the organisational outcomes as well as on the process of using our tool provided empirical validation. This iterative process led to the creation of a complete toolbox designed to help organisations raise to the knowledge transfer and utilisation challenge.

Results The toolbox includes a conceptual framework, a user's guide and two questionnaires to establish a diagnostic of the organisation's capacities of knowledge utilisation and to identify the main strategies to improve them. At the root of our toolbox is a theoretical model of organisational capacities to use knowledge, specific to health and social services organisations, that we have developed. Our model includes eight components: organisational culture, vision and leadership, knowledge acquisition, reflexivity, integration, creation and dissemination, adaptation, and relationships.

Discussion The results demonstrate that the toolbox helps decision makers to develop a common understanding of their organisation's strengths and weaknesses in terms of research knowledge utilisation capacities. Implications: The research and toolbox developed by our team provide a first step in unveiling the intricacies of knowledge utilisation in the social services sector. Eight key organisational capacities were found to be essential in facilitating and promoting knowledge utilisation in health and social services.

\section{P042 EVIDENCE-BASED GUIDELINE ON PHARMACOLOGICAL MANAGEMENT OF OSTEOARTHRITIS OF KNEE IN PRIMARY CARE SETTINGS IN HONG KONG}

M Ng, W Lam, Y Hui.

\section{0:1136/bmjqs-2013-002293.136}

Background Osteoarthritis (OA) is a common degenerative joint condition. Family physician plays an important role in the management.

Objectives The guideline seeks to assist primary health care professionals to help patients with OA knee to improve quality of life using pharmacological measures to relieve symptoms, improve drug use and reduce adverse drug incidents.

Methods The guideline is based on evidence-based literature review and was synthesised in accordance with SIGN methodology. A systematic review of literature was carried out using an explicit search strategy devised by a SIGN Information Officer. Each of the selected papers was evaluated using standard SIGH methodological checklists and the articles were subsequently reviewed. The guideline was synthesised by adaptation recommendations using the AGREE Tool. The recommendations were then modified based on the latest best available evidence. The guideline was finally reviewed internally and externally.

Results The first line drug treatment of OA knee is paracetamol in regular divided doses to a maximum of $4 \mathrm{~g} /$ day. There is good evidence to prescribe NSAID or COX-2 NSAID for reducing pain in short term. Family physician may consider prescribing weak or strong opioids with caution for moderate or severe pain. Intra-articular corticosteroid injections for short term relief can be considered. Family physician may also consider use of topical NSAIDs and hyaluronic acid.

Conclusions Use of appropriate pharmacological measures can effectively relieve symptoms, improve functions and reduce adverse drug incidents.

\section{P043 \\ FIVE MAJOR AREAS OF RESEARCH OF GRADE SYSTEM}

${ }^{1,2} \mathrm{Y}$ Chen, ${ }^{1,2} \mathrm{~L}$ Yao, ${ }^{1,2} \mathrm{Q}$ Wang, ${ }^{1,2} \mathrm{Q}$ Wu, ${ }^{1,2} \mathrm{X}$ Wang, ${ }^{1,2} \mathrm{~K}$ Yang. ${ }^{1}$ Evidence-Based Medicine Center of Lanzhou University, Lanzhou, China; ${ }^{2}$ Chinese GRADE Center, Lanzhou, China

\section{0:1136/bmjqs-2013-002293.137}

Background Over the past 10 years, more and more guideline developers and systematic review authors adopt the GRADE method. Also, many researchers and groups become interested in discussing, studying and developing it.

Methods We searched and analysed all GRADE papers published in academic journals from 2004 to 2012, abstracts in Cochrane colloquium from 2000 to 2012 and G-I-N Conferences from 2003 to 2012, attended GRADE workshops and meeting in GIN and Cochrane colloquium.

Results We found the following five major areas of research of GRADE system: 1. Comparison and Trend Analysis between GRADE and other different rating systems 2. Reasons analysis for downgrading and upgrading the quality of evidence and 